\title{
Kommunikationsmodelle für das Tagesgeschäft in der Unternehmenspraxis
}

\author{
Kommunikation im Unternehmensalltag wird zunehmend komplexer. Um \\ die alten und neuen Herausforderungen, von Kommunikationsstörungen \\ über neue Kommunikationskanäle und bis hin zu Social Media, besser zu \\ begreifen, können Kommunikationsmodelle helfen.
}

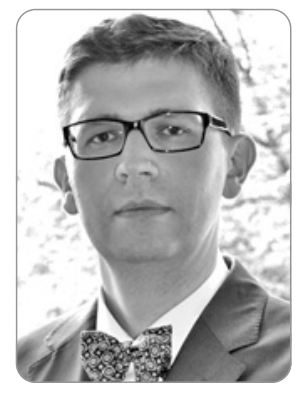

Prof. Dr. Olexiy Khabyuk

ist berufener Hochschullehrer für das Gebiet BWL, insbesondere Kommunikation und Multimedia, an der Hochschule Düsseldorf. Bevorzugte Forschungsgebiete: Ganzheitliche Betrachtung von neuen Mediengattungen als Geschäftsmodelle, Kommunikationsmittler und Faktoren der gesellschaftlichen Meinungsbildung, Entwicklung von Kommunikationsberufffeldern.

Stichwörter: Kommunikationsstörungen, Individualkommunikation, Kommunikationskanäle, computervermittelte Kommunikation, Social Media

\section{Schöne neue Welt der Kommunikation}

Der Berufsalltag ist vermeintlich einfacher geworden: Flachere Hierarchien, laxere Bekleidungsordnung, niemand achtet mehr auf Rechtschreibfehler in Geschäftsmails. Doch diese schöne neue Welt ist trügerisch.

Aufgrund der zunehmenden Technisierung der Kommunikation entstehen immer neuere Kommunikationskanäle in immer kürzerer Zeit. Während zwischen der Entstehung des Buchdrucks (1450), der Zeitung (1650) des Telegraphs (1883) jeweils ca. 200 Jahre vergangen sind, liegen zwischen dem ersten markttauglichen Heimcomputer (1982) und dem internetbasierten Sprachassistenten Amazon Echo (2015) gerade mal 33 Jahre (vgl. Khabyuk, 2019, S. 28). Immer neuere Kommunikationskanäle (Facebook, WhatsApp, Instagram, Snapchat, TikTok etc.) sprießen aus dem Boden und befeuern uns mit einem schier unendlichen Strom an Botschaften. Während man früher im Büro telefonisch, per Fax und per Brief erreichbar sein musste, kommt heute eine E-Mail-Flut auf uns zu, die es nach Erwartung des Senders innerhalb von 24 Stunden zu bearbeiten gilt. Auch die Arbeitgeber erwarten eine ständige Erreichbarkeit und zeitliche Flexibilität, wenn schon nicht per Mobiltelefon, dann zumindest per E-Mail. Und dann kam 2018 noch die DSGVO... Während früher vor allem Berufe im Marketing und PR besonders kommunikationsstark sein mussten, gilt die neue Kommunikationsrealität heute für nahezu alle Geschäftsteilnehmer.

Was verstehen wir im Folgenden unter Kommunikation? Es gibt zahlreiche Definitionsversuche. Prägnant und bis heute akzeptiert beschreibt Lasswell (1964 (1948), S. 37) den Akt der Kommunikation mit einer fünfteiligen Frage: (1) Wer (2) sagt was (3) in welchem Kanal (4) zu wem und (5) mit welchem Effekt? Sicherlich gibt es eine Reihe an präziseren Definitionen, für unsere Zwecke ist diese aber völlig ausreichend.

In Anlehnung an Maletzke (1963, S. 21-32) unterscheiden wir ferner zwischen Individual- und Massenkommunikation. Individualkommunikation verläuft dabei meist direkt, zweiseitig und grundsätzlich mit einem eng definierten Personenkreis (z.B. z.B. Gespräche/Chat mit Freunden). Massenkommunikation dagegen ist stets indirekt, einseitig und richtet sich an ein öffentliches Publikum (z.B. TV-Spot). Für die Massenkommunikation ist der Einsatz von Medienkanälen eine Voraussetzung.

Die obigen Definitionen sind in der Zeit elektronischer Massenmedien entstanden, die in der Regel eine 0ne-toMany-Beziehung ohne einen Rückkanal zugelassen haben (z.B. klassische Medien wie TV, Radio, Printzeitung). Zum damaligen Zeitpunkt war Massenkommunikation mit der Medienkommunikation gleichzusetzen. Individualkommunikation fand in der Regel als Direktkommunikation statt und war nur mit wenigen Ausnahmen medienvermittelt, z.B. per Telefon oder Brief.

Die heutigen computervermittelten digitalen Medien dagegen bauen (über Internetprotokolle) One-to-one-Beziehungen mit einem vollwertigen Rückkanal auf. Damit unterstützen sie gleichermaßen Individual- und Massenkommunikation. Dies hat einerseits dazu geführt, dass ein Großteil der Individualkommunikation heute nicht mehr direkt, sondern über digitale Medien stattfindet und sich somit die Art der Individualkommunikation verändert. An- 
Quellen: Verkürzt nach Khabyuk, 2019, S. 40, in Anlehnung an Six/Gleich/Gimmler, 2007, S. 25.

Tab. 1: Abgrenzung der Individual- vs. Massenkommunikation und Direkt- vs. Medienkommunikation

\begin{tabular}{|c|c||c|}
\hline Direktkommunikation & \multicolumn{2}{|c|}{ Medienkommunikation } \\
\hline \multicolumn{2}{|c|}{ Individualkommunikation } & Massenkommunikation \\
\hline $\begin{array}{c}\text { Direkte interpersonale } \\
\text { Kommunikation }\end{array}$ & $\begin{array}{c}\text { Computer-/medienvermittelte } \\
\text { individuelle Kommunikation }\end{array}$ & \\
\hline $\begin{array}{c}\text { Watzlawick/Beavin/Jackson, } \\
2011\end{array}$ & Daft/Lengel, 1984 & $\begin{array}{c}\text { Lazarsfeld/Berelson/Gaudet, } \\
1968\end{array}$ \\
\hline
\end{tabular}

dererseits ist eine von den Plattformbetreibern bewusst herbeigeführte Vermischung der Individual- und Massenkommunikation zu beobachten (z.B. auf Instagram, Facebook), um den massenkommunikativen Botschaften die Glaubwürdigkeit von individualkommunizierten Botschaften zu verleihen. Im Tagesgeschäft der Unternehmenspraxis sind vor allem Phänomene der Individualkommunikation relevant, woran sich die in diesem Aufsatz besprochenen Modelle orientieren. Auch die "neue“ Massenkommunikation soll am Rande thematisiert werden (siehe Tab. 1).

\section{Die Regeln der zwischenmenschlichen}

Kommunikation nach Watzlawick/Beavin/Jackson, 2011

Wodurch ist zwischenmenschliche Kommunikation gekennzeichnet? Watzlawick/Beavin/Jackson (2011, erstmalig veröffentlicht in 1967) führen psychische Störungen auf Verletzung der Regeln der Kommunikation zurück. Sie argumentieren, dass Menschen ihre Realität durch Kommunikation konstruieren. Aus der gestörten Kommunikation folgen psychische Störungen. Die Autoren stellen Regeln (Axiome) für eine ungestörte Kommunikation auf. Die fünf Axiome bauen aufeinander auf (S. 57-81). Wir werden ferner versuchen, zu jedem Axiom ein Kommunikationsgebot zu formulieren (vgl. Khabyuk, 2019, S. 64-71).

1. „Man kann nicht nicht kommunizieren.“ Mit anderen Worten: Alles, was man tut, kann als Kommunikation gewertet werden. Da das Verhalten kein Gegenteil hat, kann selbst das stumme Vorbeilaufen eines Kollegen auf dem Flur als eine Kommunikationsbotschaft mehrdeutig gewertet werden - als bewusstes Ignorieren oder als stressbedingte Handlung. Auch können verschränkte Arme bei einer Präsentation Ablehnung, Angst oder lediglich Rückenschmerzen, Kältegefühl u.Ä. bedeuten. Das führt uns zum folgenden Kommunikationsgebot: Man sollte stets versuchen, sein eigenes Verhalten eindeutig zu artikulieren und mehrdeutiges Kommunikationsverhalten von Kommunikationspartnern bedächtig zu interpretieren.

2. Jede Botschaft besteht aus zwei Teilen - einem Beziehungs- und einem Inhaltsaspekt. Dabei ist Beziehung das wichtigere Element und gibt den Kontext für den Inhalt vor. Wenn beispielsweise Titel, Anrede, Namen in einem Kundenbrief falsch geschrieben oder weggelassen werden, kann dies die Beziehung beschädigen und der
Kunde wird den ansonsten fehlerfreien Inhalt ignorieren. Das gleiche trifft auf Botschaften $z u$, die in einer falschen Intonation ausgesprochen werden. Auch in einer wissenschaftlichen Arbeit kann die Missachtung der Rechtschreibung/Formatierung die Beziehung des Studierenden zum Betreuer beschädigen. Mögliche Folge: Die Arbeit wird nicht mehr sachlich gelesen. Auch nach dem bekannten Harvard-Prinzip (vgl. Fischer/Patton/Ury, 2004) sind langfristige Verhandlungserfolge nur mit einer intakten Beziehung möglich. Ist beispielsweise die Arbeitsatmosphäre stark gestört, sollte man einen betriebsfremden Moderator einsetzen, um wieder zur sachlichen Ebene zurückzukehren. Und nicht zuletzt in Scrum-Projekten ist mindestens ein retrospektives Meeting eingeplant, bei dem Beziehungen geklärt werden können. Daran orientiert sich auch unser Kommunikationsgebot: Jede Mitteilung sollte vor dem Hintergrund der Beziehung zum Kommunikationspartner formuliert werden, wobei die Mitteilungsform ausschlaggebend für das Verständnis der Mitteilung ist.

3. Beziehungen entstehen, indem einzelne Kommunikationsabläufe seitens der Kommunikationspartner subjektiv bewertet werden. Dabei kann die gleiche Botschaft völlig unterschiedlich interpretiert werden, z.B. kann die Führungskraft den Mitarbeiter immer stärker unter Druck setzen, weil er passiv ist. Der Mitarbeiter ist aber gerade deshalb passiv geworden, weil die Führungskraft ihn ständig angreift. Man kann dieses Beispiel leicht für interdisziplinäre und internationale Teams weiterentwickeln. Das Kommunikationsgebot lautet deshalb: Versuche die Verhaltensregeln des Kommunikationspartners $\mathrm{zu}$ erkennen und ein gemeinsames Verständnis der Dinge herauszuarbeiten, z.B. in Form von geschriebenen und ungeschriebenen Regeln, Prozessen, Ritualen.

4. Für die Beziehungskommunikation sollten eher nonverbale Kommunikationsmodi (in der Sprache der Autoren - analoge) verwendet werden, für die Sachkommunikation eher verbale (in der Sprache der Autoren - digitale). Analoge Kommunikation ist jedoch eher unpräzise (denken Sie an Pantomime). Digitale Kommunikation, wie geschriebener Text, ist wiederum für Beziehungsbotschaften eher ungeeignet (z.B. sind schriftlich ausformulierte Unternehmensleitlinien ohne eine gelebte Führungskultur Makulatur). Kommunikationsgebot: 


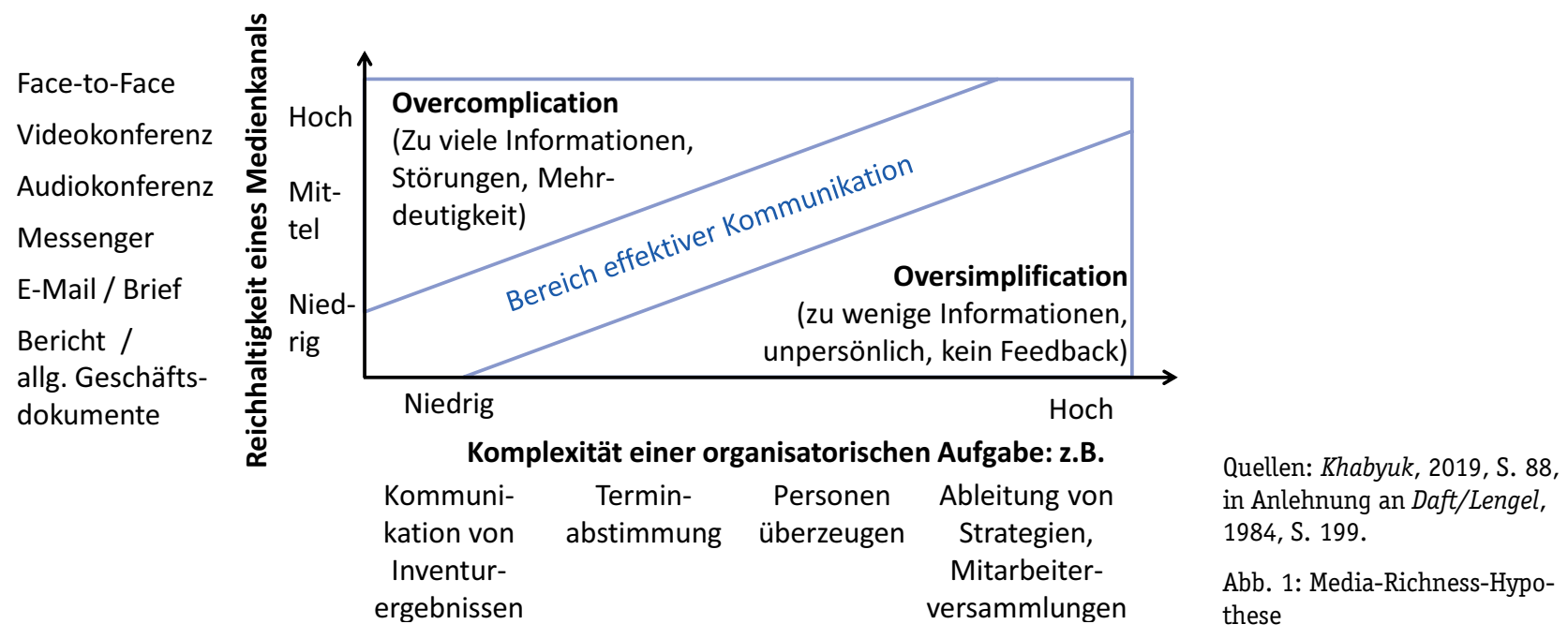

Es sollte daher darauf geachtet werden, welche Botschaften über welche Modi und die sie unterstützenden Kanäle $\mathrm{zu}$ kommunizieren sind (z.B. sollte man die Teambildung bei räumlich verteilten Teammitgliedern zumindest am Anfang über eine persönliche Aktivität unterstützen).

5. Die Beziehung zwischen den Kommunikationspartnern ist entweder symmetrisch oder komplementär, d.h. entweder auf Gleichheit oder auf Unterschiedlichkeit beruhend. Beispielsweise besteht zwischen Arzt und Patient eine eindeutige komplementäre Beziehung. Verweist der Patient allerdings auf eine eigene internetrecherchierte Diagnose, kann das zu einem Konflikt führen. Wenn die Erwartungen an die Beziehungsart unterschiedlich sind, besteht häufig großes Konfliktpotenzial, z.B. zwischen Eltern (komplementär) und Kindern (symmetrisch), Dozenten (komplementär) und Studierenden der Generation Y (symmetrisch), Führungskraft in Deutschland (symmetrisch) und Mitarbeiter in China (komplementär) etc. Als Kommunikationsgebot kann man hier lediglich anraten, die eigenen Erwartungen mit den Erwartungen des Kommunikationspartners abzugleichen.

\section{Die Reichhaltigkeit von Kommunikationskanälen nach Daft/Lengel, 1984}

Während die obigen Axiome vornehmlich für die Individualkommunikation ohne Medieneinsatz formuliert wurden, haben sich Daft/Lengel (1984) im Auftrag der US-Marine die Frage gestellt, welche Kommunikationskanäle sich zur Lösung welcher Probleme in Unternehmen eignen. Die Hauptmerkmale komplexer Problemlagen in Unternehmen seien ihrer Meinung nach vorrangig die Mehrdeutigkeit von $\mathrm{zu}$ verarbeitenden Informationen (Schwierigkeit, ein Problem eindeutig zu interpretieren, z.B. Erklärung des Rückgangs von Absatzzahlen) und etwas nachrangig die
Unsicherheit (unzureichende Information über einen Sachverhalt, der keiner Interpretation bedarf, z.B. aktuelle Lagerbestände). Die damaligen textbasierten Informationssysteme konnten sehr wohl eine schnelle Unsicherheitsreduktion durch entsprechende Reports und effiziente abteilungsübergreifende Datenübergabe sicherstellen (z.B. in ERP-Systemen). Um dagegen ein mehrdeutiges Problem zu lösen, bedurfte es reichhaltigerer Medien, so die Autoren.

Die Medien sind umso reichhaltiger, je mehr Kommunikationsmodi (mündliche Sprache, paraverbale Signale, Körpersprache, Zeichen/geschriebene Sprache), je schnelleres Feedback und je persönlichere Bezüge sie unterstützen. So ist Face-to-Face-Kommunikation am reichhaltigsten, ein allgemeiner Report weist jedoch die geringste Reichhaltigkeit auf.

Die Autoren haben dann ihre bis heute anerkannte Hypothese formuliert, dass je komplexer (d.h. vor allem - mehrdeutiger) ein Problem ist, desto reichhaltiger sollte der Kommunikationskanal sein, der zur Lösung herangezogen wird (visualisiert in $A b b .1$ ). So sollte ein Change-Prozess in einem Unternehmen in erster Linie über persönliche Gespräche initiiert werden und kann erst später durch allgemeine Texte im Intranet unterstützt werden. Für bestimmte eindeutige Probleme, z.B. zur Kommunikation von Inventurergebnissen sollten dementsprechend aber auch informationsärmere Kanäle verwendet werden, z.B. schriftliche Reports.

Missachtet man das in Abb. 1 skizzierte Verhältnis der effektiven Kommunikation, würde dies entweder zu einer unsachgemäßen Vereinfachung des Problems und folglich Fehlentscheidungen („oversimplification“) führen oder auch zu einem ineffizienten Umgang mit Kommunikationsressourcen ("overcomplication“). Man denke hier an einen lästigen Mitarbeiter, der anstatt eine E-Mail zu schreiben der Führungskraft jede Kleinigkeit mündlich mitzuteilen versucht. Es gibt zahlreiche andere Beispiele für eine inadäquate Verwendung von Kommunikationskanälen, z.B. 
das Verfassen von seitenlangen unstrukturierten E-Mails bei diskussionsbedürftigen Themen (besser: Telefonkonferenz), die Nutzung von WhatsApp in der kreativen Phase einer Gruppenarbeit an der Hochschule (besser: persönliches Gruppenmeeting) etc. Andererseits wurden elektronische Tools dafür kritisiert, dass sie zu wenig emotionale Aspekte übertragen haben. Inzwischen lässt sich dieser Kritikpunkt durch den unterstützenden Einsatz von Emojis und anderen multimedialen Inhalten entkräften. Beispielsweise werden selbst Vorstellungsgespräche mitunter per Videokonferenz durchgeführt. Von daher ist der in Abb. 1 visualisierte Zusammenhang sehr allgemein und sollte in der Praxis stets einzelfallbezogen betrachtet werden. Wir erwarten, dass die Corona-diktierte Home-Office-Zeit die Gewohnheiten in der geschäftlichen Kommunikation massiv zugunsten digitaler Kanäle verschieben wird.

\section{Brauchen Unternehmen Social Media? Meinungsfüh- rerforschung nach Lazarsfeld/Berelson/Gaudet, 1968}

Um das Verwischen von Individual- und Massenkommunikation in den digitalen Medien zu umschreiben, wird ein früher Beitrag zur Meinungsbildung im Vorfeld der politischen Wahlen von Lazarsfeld/Berelson/Gaudet (1968, zuerst publiziert in 1944) herangezogen. Die darin formulierte Hypothese zum Zwei-Stufen-Fluss (S. 150 ff.) kann auch die Wirkungsweise der Social Media erklären (visualisiert in $A b b .2$ ). Nach dieser Hypothese wirken die massenmedial vermittelten Inhalte persuasiver Art (z.B. Wahlprogramm, Produktinformationen) nicht direkt auf die Konsumenten ein, sondern zunächst auf die Meinungsführer (Massenkommunikation). Meinungsführer wiederum üben einen direkten Einfluss auf die ihnen bekannten Gruppenmitglieder in persönlichen Gesprächen aus (Individualkommunikation). Dabei gibt es Meinungsführer in jeder sozialen Schicht. Sie zeichnen sich durch einen starken Medienkonsum und eine besondere Diskussionsfreude aus. Auch wenn in späteren Studien die empirische Überprü- fung der Hypothese versagt, so ist doch die Augenscheinvalidität des Modells sehr hoch. Denn wenn es um Wahlentscheidungen geht, so lassen wir uns doch sehr gerne von Meinungsführern aus unserem persönlichen Umfeld (Freunden, Familienmitgliedern etc.) beeinflussen, die auf uns besonders gut informiert wirken.

Die Influencer-Kommunikation in Social Media funktioniert ähnlich. Influencer sind Personen, die eine besondere Affinität für neue Produkte aufweisen und den Konsum im Kreis ihrer „Freunde” zelebrieren. Dabei findet diese Anschlusskommunikation nicht wie im Ursprungsmodell direkt, sondern über Social Media statt. Der Individualitätsgrad der Botschaften hängt von der Follower-Zahl ab. Auch Ihre Freunde, die eine Produktinformation über Facebook teilen, schlüpfen in die Influencer-Rolle. Influencer mit Millionen von Followern (wie z.B. Dagibee) dagegen gaukeln Individualkommunikation lediglich vor, auch wenn sie auf einige Kommentare der Follower reagieren. Botschaften von Influencern (Earned Content) sind deshalb wirksamer als Botschaften aus klassischen Bezahlmedien (Paid Content), weil Individualkommunikation eher unserer natürlichen Kommunikationsart entspricht. Dazu kommt, dass die gleichen Plattformen tatsächlich für Individualkommunikation genutzt werden (z.B. Facebook Messenger).

Vor allem Digital Natives, die mit solchen Plattformen aufgewachsen sind, können nicht mehr trennscharf zwischen Individual- und Massenkommunikation unterscheiden. Es ist ihr gewöhnlicher „modus communicandi“. Und so verwundert es nicht, wenn Unternehmen Mitarbeiter auffordern, das eigene Unternehmen in den privaten Social-Media-Präsenzen zu bewerben und so zu Influencern zu werden, indem sie z.B. Stellenangebote teilen etc. Durch das Teilen solcher Botschaften und anderer Unternehmensmitteilungen transformieren Nutzer Massenkommunikation in Individualkommunikation.
Quellen: Khabyuk, 2019, S. 137, in Anlehnung an Emrich, 2008, S. 68.

Abb. 2: Zwei-Stufen-Fluss-Hypothese nach Lazarsfeld/Berelson/ Gaudet, 1968

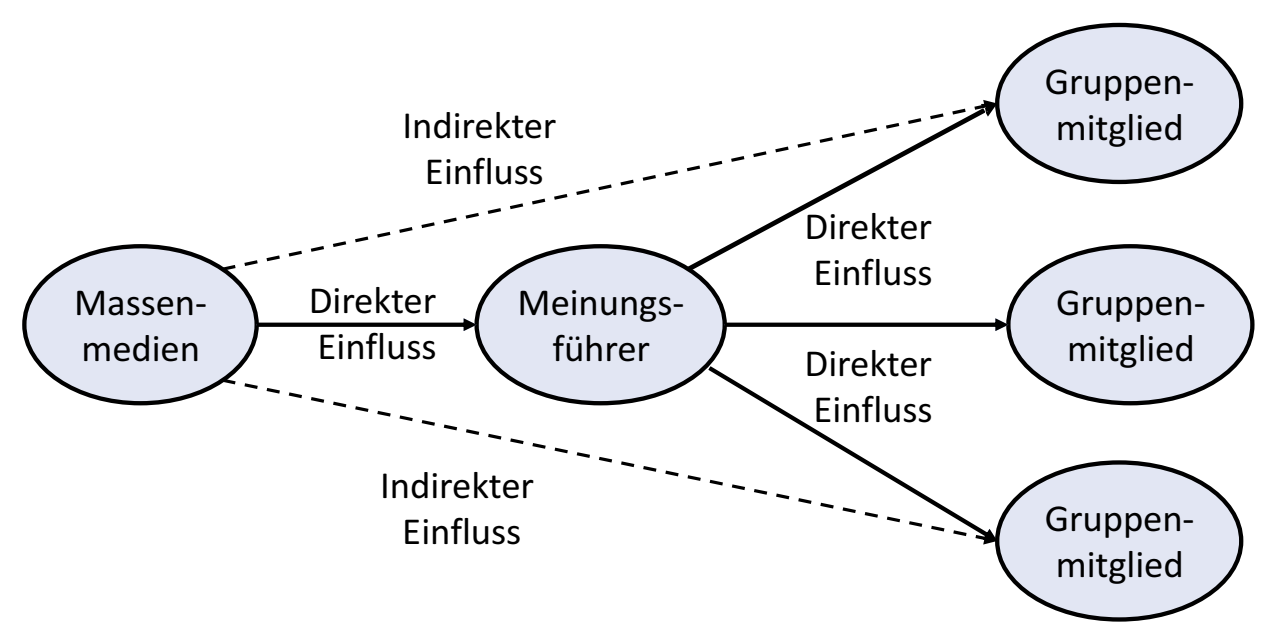

